\title{
CIÊNCIA'NATURA
}

\section{Simulação da dispersão de poluentes em um modelo Euleriano considerando coeficientes de difusão dependentes da distância da fonte e o fenômeno de meandro do vento}

Pollutants dispersion simulation in an Eulerian model considering eddy diffusivities that depend of the source distance and the wind meandering phenomenon

${ }^{1}$ Viliam Cardoso da Silveira, ${ }^{1}$ Gervásio Annes Degrazia e ${ }^{2}$ Daniela Buske

${ }^{1}$ UFSM - Universidade Federal de Santa Maria, RS, Brasil

${ }^{2}$ UFPel - Universidade Federal de Pelotas, RS, Brasil

\section{Resumo}

O objetivo deste trabalho é simular a dispersão de poluentes em um modelo Euleriano considerando o fenômeno de meandro do vento e coeficientes de difusão dependentes da distância da fonte. O modelo de dispersão de poluentes resolve analiticamente a equação de advecção-difusão pelo método 3D-GILTT. Para considerar o meandro do vento no modelo de dispersão decompomos o vento nas componentes u e v e calculamos as funções de autocorrelação e espectros do vento. Os resultados mostram uma boa concordância entre as concentrações observadas e previstas.

Palavras-chave: Dispersão de poluentes, equação de advecção-difusão, método 3D-GILTT.

\section{Abstract}

The aim of this work is to simulate the pollutants dispersion in an Eulerian model considering the wind meandering phenomenon and eddy diffusivities in terms of source distance. The pollutants dispersion model solve analytically the adcevction-diffusion equation by 3D-GILTT technique. To consider the wind meandering in the dispersion model, we decompose the wind in the $u$ and $v$ components and we calculate the autocorrelation functions and wind spectra. The results show a good agreement between the observed and simulated concentrations.

Keywords: Pollutants dispersion, advection-diffusion equation, 3D-GILTT technique. 


\section{Introdução}

Comumente, em condições estáveis na Camada Limite Planetária (CLP), durante situações nas quais a velocidade do vento apresenta baixa magnitude $(U \leq 1,5 \mathrm{~m} / \mathrm{s})$, são observadas oscilações de baixa frequência do vento horizontal Anfossi et al. (2005). Essas oscilações direcionais do vento horizontal são conhecidas como fenômeno de meandro do vento. Segundo Mortarini et al. (2013); Mortarini e Anfossi (2015), deve ser observados dois critérios complementares para considerar a ocorrência de meandro, além da condição de vento fraco. O primeiro é que a taxa (em módulo) entre os parâmetros de ajuste da função de autorrelação deve ser maior ou igual a 1 e o segundo critério é verificar a existência de lóbulos negativos na função de autocorrelação.

Conforme Mortarini e Anfossi (2015), em condições de vento fraco, a função de autocorrelação Euleriana das componentes do vento horizontal tem um comportamento oscilatório característico identificando uma frequência de meandro bem definida e associada com essa frequência o espectro tem um pico distinto que caracteriza o meandro do vento.

A função de autocorrelação mede o grau de correlação de uma variável consigo mesma em um dado instante de tempo e em um instante de tempo posterior. Ela é calculada com base na seguinte expressão

$$
r(k)=\frac{\sum_{i=1}^{n-k}\left(x_{i}-\bar{x}\right)\left(x_{i+k}-\bar{x}\right)}{\sum_{i=1}^{n}\left(x_{i}-\bar{x}\right)^{2}}
$$

sendo que $n$ é o número total de experimentos $(0 \leq k \leq n-1)$ e $x$ refere-se a um dado experimento.

A partir da função de autocorrelação, calculada com base nos dados observados, calcula-se o ajuste. O método de ajuste utilizado é o algoritmo de Gauss-Newton Bates e Watts (1988). Este método consiste em ajustar um modelo não linear, resolvendo desta maneira, um problema linear de mínimos quadrados Departamento de computação e matemática (2015).

Dentre as fórmulas de ajuste mais utilizadas, pode-se citar a proposta por Frenkiel Frenkiel (1953)

$$
r(\tau)=e^{-p \tau} \cos (q \tau)
$$

e a proposta por Szinvelski Szinvelski et al. (2013)

$$
r(\tau)=\frac{\cos (q \tau)}{(1+p \tau)^{2}}
$$

sendo $p$ e $q$ os parâmetros de ajuste.

Geralmente, os modelos Eulerianos de dispersão de poluentes consideram que o vento médio é dominante na direção x e desprezam a componente $\mathrm{v}$ do vento Buske et al. (2007). No caso da ocorrência de meandro, as duas componente horizontais (u e v) do vento devem ser consideradas. Assim, o objetivo desse trabalho é avaliar a dispersão de poluentes na atmosfera, considerando o fenômeno de meandro do vento que ocorre em condições de vento fraco.

\section{Metodologia}

A equação de advecção-difusão parametrizada Blackadar (1997) é escrita como

$$
\bar{u} \frac{\partial \bar{c}}{\partial x}+\bar{v} \frac{\partial \bar{c}}{\partial y}+\bar{w} \frac{\partial \bar{c}}{\partial z}=\frac{\partial}{\partial x}\left(K_{x} \frac{\partial \bar{c}}{\partial x}\right)+\frac{\partial}{\partial y}\left(K_{y} \frac{\partial \bar{c}}{\partial y}\right)+\frac{\partial}{\partial z}\left(K_{z} \frac{\partial \bar{c}}{\partial z}\right)
$$

Os termos do lado esquerdo da equação 4 representam a advecção e os termos do lado direito à difusão. As componentes médias $\bar{u}$ e $\bar{v}$ do vento foram calculadas da seguinte forma

$$
\begin{aligned}
& \bar{u}=V \operatorname{sen}(\theta) \\
& \bar{v}=V \cos (\theta)
\end{aligned}
$$

sendo $V$ a velocidade do vento e $\theta$ a direção do vento. As condições de contorno da equação 4 são

$$
K_{x} \frac{\partial \bar{c}\left(L_{x}, y, z\right)}{\partial x}=K_{y} \frac{\partial \bar{c}(x, 0, z)}{\partial y}=K_{y} \frac{\partial \bar{c}\left(x, L_{y}, z\right)}{\partial y}=K_{z} \frac{\partial \bar{c}(x, y, 0)}{\partial z}=K_{z} \frac{\partial \bar{c}(x, y, h)}{\partial z}=0
$$

A condição de fonte é $\bar{u} \bar{c}(0, y, z)=Q \delta\left(y-y_{o}\right) \delta\left(z-H_{s}\right)$, sendo $Q$ a taxa de emissão da fonte $(g / s), h$ a altura da camada limite planetária, $H_{s}$ a altura da fonte $(m), L_{x}$ e $L_{y}$ os limites para longe da fonte no eixo $x$ e $y$, respectivamente $(m)$ e $\delta$ é a função delta de Dirac.

Assumindo que a velocidade $\bar{w}$ é nula e ainda que o coeficiente de difusão $K_{y}$ tem dependência somente na direção z $\left(K_{y}^{\prime}=0\right)$ na equação 4 , a seguinte equação é obtida

$$
-\bar{u} \frac{\partial \bar{c}(x, y, z)}{\partial x}-\bar{v} \frac{\partial \bar{c}(x, y, z)}{\partial y}+\frac{\partial}{\partial x}\left(K_{x} \frac{\partial \bar{c}(x, y, z)}{\partial x}\right)+K_{y} \frac{\partial^{2} \bar{c}(x, y, z)}{\partial y^{2}}+\frac{\partial}{\partial z}\left(K_{z} \frac{\partial \bar{c}(x, y, z)}{\partial z}\right)=0
$$


A equação de advecção-difusão é resolvida pela combinação das técnicas da transformada de Laplace e GILTT (Generalized Integral Laplace Transform Technique) Buske et al. (2007); Moreira et al. (2009); Buske et al. (2012). Essa técnica da transformada integral combina uma expansão em série com uma integração. Na expansão, é usada uma base trigonométrica determinada com a ajuda de um problema auxiliar de Sturm-Liouville. A integração é feita em todo intervalo da variável transformada, fazendo uso da propriedade de ortogonalidade da base usada na expansão. O sistema de equações diferenciais ordinárias resultante é analiticamente resolvido usando a transformada de Laplace e diagonalização Buske et al. (2012).

Como a altura de medida do poluente é diferente da altura de emissão, utilizamos lei de potência do vento Panofsky e Dutton (1984).

$$
\frac{\bar{V}}{\overline{V_{1}}}=\left(\frac{z}{z_{1}}\right)^{0,1}
$$

no qual $\bar{V}$ e $\bar{V}_{1}$ são as velocidades médias horizontais do vento nas alturas $z$ e $z_{1}$.

Para validar o modelo foram utilizados dados de vento fraco reportados no experimento INEL (USA) Sagendorf e Dickson (1974). O experimento INEL consiste de uma série de testes difusivos, conduzidos em um terreno plano e uniforme. O poluente $S F_{6}$ foi coletado em arcos posicionados nos raios de 100,200 e $400 \mathrm{~m}$ do ponto de emissão na altura de $0,76 \mathrm{~m}$ do solo. O poluente foi liberado de uma altura de $1,5 \mathrm{~m}$ acima do nível do solo. O parâmetro meteorológico vento em $2 \mathrm{~m}$ foi obtido do experimento. A rugosidade na superfície foi de $0,005 m$.

Os índices estatísticos são usados para avaliar o desempenho do modelo em representar os dados observados. São avaliados, respectivamente, os índices estatísticos, desvio quadrático médio normalizado (NMSE), coeficiente de correlação (COR) e desvio fracional padrão (FS) Hanna (1989).

\section{Parametrização da turbulência}

Para representar a difusão perto da fonte em condições de vento fraco, os coeficientes de difusão devem ser considerados como funções não somente da turbulência (ou seja, do comprimento dos grandes turbilhões e escala de velocidade), mas também da distância da fonte. Seguindo essa idéia, Degrazia et al. (1996) propuseram a seguinte formulação para os coeficientes de difusão $\left(K_{\alpha}\right)$, em condições estáveis, levando em conta o efeito de memória da pluma de poluentes ( $\alpha$ é para $x, y$ e $z$ )

$$
K_{\alpha}=\frac{2 \sqrt{\pi} 0,64 u_{*} h a_{i}^{2}(1-z / h)^{\alpha_{1}}(z / h) X^{*}\left[2 \sqrt{\pi} 0,64 a_{i}^{2}(z / h)+8 a_{i}\left(f_{m}\right)_{i}(1-z / h)^{\alpha_{1} / 2} X^{*}\right]}{\left[2 \sqrt{\pi} 0,64(z / h)+16 a_{i}\left(f_{m}\right)_{i}(1-z / h)^{\alpha_{1} / 2} X^{*}\right]^{2}}
$$

em que $i=u, v, w, X^{*}$ é a distância adimensional $\left(X^{*}=x u_{*} /(\bar{u} h)\right)$, $h$ é a altura da CLE, $\alpha_{1}$ é uma constante que depende da evolução da CLE, $\left(f_{m}\right)_{i}$ é a frequência do pico espectral dada por

$$
\left(f_{m}\right)_{i}=\left(f_{m}\right)_{n, i}\left(1+3,7 \frac{z}{\Lambda}\right)
$$

sendo $\left(f_{m}\right)_{n, i}$ a frequência do pico espectral na estratificação neutra $\left[\left(f_{m}\right)_{n, w}=0,33 ;\left(f_{m}\right)_{n, v}=0,16 ;\left(f_{m}\right)_{n, u}=0,045\right]$, $a_{u}=3889, a_{v}=1094$ e $a_{w}=500$ e $f_{c}=10^{-4} s^{-1}$ é o parâmetro de Coriolis, $\mathrm{z}$ a altura acima do solo e $\Lambda$ o comprimento de Monin-Obukhov local dado pela seguinte formulação

$$
\Lambda=L\left(1-\frac{z}{h}\right)^{\left(1,5 \alpha_{1}-\alpha_{2}\right)}
$$

em que $\left[\alpha_{1}=1,5 ; \alpha_{2}=1\right]$ e $a_{i}$ é dado por $a_{i}=\left(2,7 c_{i}\right)^{1 / 2} /\left(f_{m}\right)_{n, i}^{1 / 3}$, sendo que $c_{v, w}$ e $c_{u}$ são dados respectivamente por $\left[c_{v, w}=0,4 ; c_{u}=0,3\right]$.

Os coeficientes de difusão nas três direções (longitudinal, lateral e vertical) que também levam em conta o efeito de memória da pluma de poluentes, podem ser escritos da seguinte forma Sorbjan (1989)

$$
K_{\alpha}=\frac{\sigma_{i}^{2} t}{\left(1+\frac{t}{2 T_{L i}}\right)^{2}}\left(1+\frac{t}{4 T_{L i}}\right)
$$

Para obtermos os coeficientes em cada direçõo, precisamos das variâncias e escalas de tempo de decorrelação naquela direçõo, ou seja, as variâncias nas direções $x, y$ e $z$, respectivamente, são dadas por

$$
\begin{aligned}
& \sigma_{u}^{2}=6,0\left(1-\frac{z}{h}\right)^{2} u_{*}^{2} \\
& \sigma_{v}^{2}=3,3\left(1-\frac{z}{h}\right)^{2} u_{*}^{2}
\end{aligned}
$$




$$
\sigma_{w}^{2}=2,5\left(1-\frac{\sim}{h}\right)^{-} u_{*}^{2}
$$

Já as escalas de tempo de decorrelação nas direções longitudinal, lateral e vertical são dadas, respectivamente, por

$$
\begin{aligned}
& T_{L u}=\frac{z k}{6,0\left[1+4,7\left(\frac{z}{L}\right)\right]\left(1-\frac{z}{h}\right) u_{*}} \\
& T_{L v}=\frac{z k}{3,3\left[1+4,7\left(\frac{z}{L}\right)\right]\left(1-\frac{z}{h}\right) u_{*}} \\
& T_{L w}=\frac{z k}{2,5\left[1+4,7\left(\frac{z}{L}\right)\right]\left(1-\frac{z}{h}\right) u_{*}}
\end{aligned}
$$

\section{Resultados}

A Figura 1 mostra o lóbulo negativo observado por meio da função de autocorrelação calculada com base na componente u do vento dada pela fórmula 5. Para gerar essa figura foi feito um loop no ângulo de vento de 0 a 360 graus. A razão (em módulo) entre os parâmetros de ajuste proposto por Frenkiel é igual a 7,728815 e proposto por Degrazia igual a 12,54798 . O período de meandro do ajuste proposto por Frenkiel é $1.869,924 \mathrm{~s}$, já o período de meandro do ajuste proposto por Degrazia é $1.873,425 \mathrm{~s}$. Além do mais, observa-se um pico no espectro na baixa frequência o que provavelmente se refere ao fenômeno de meandro do vento.

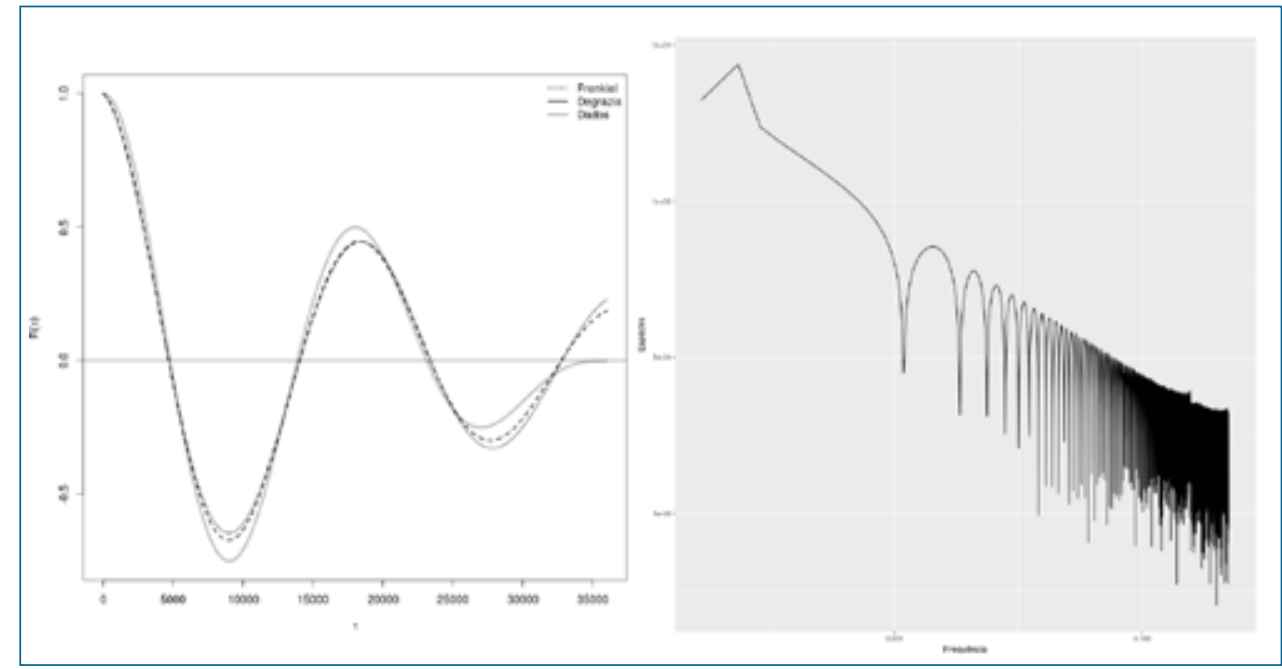

Figura 1: Função de autocorrelação calculada com base na componente u do vento.

A Figura 2 mostra a presença do lóbulo negativo observado por meio da função de autocorrelação calculada com base na componente $\mathrm{v}$ do vento dada pela fórmula 6. Aqui também foi feito um loop no ângulo do vento. A razão entre os parâmetros de ajuste proposto por Frenkiel é igual a 8,09196 e proposto por Degrazia igual a 12,96058. O período de meandro do ajuste proposto por Frenkiel é $1.745,296$ s, já o período de meandro do ajuste proposto por Degrazia é 1.746,364 s. Nesse caso também observa-se um pico no espectro na baixa frequência.

A presença de lóbulos negativos observados nas funções de autocorrelação, juntamente com a razão dos parâmetros de ajuste maior do que 1 , conforme a literatura, indicam a presença do fenômeno de meandro do vento. Observa-se também picos no espectro na baixa frequência o que provavelmente se refere ao fenômeno de meandro do vento. Assim, com base nas Figuras 1-2, pode-se concluir que o presente modelo Euleriano é capaz de simular o meandro do vento, que se refere as oscilações de baixa frequência.

Agora que já mostramos que o modelo é capaz de capturar as características observadas para o fenômeno de meandro do vento, vamos fazer algumas simulações com o modelo de dispersão já configurado para essas situações, testando duas parametrizações para os coeficientes de difusão. A Tabela 1 mostra os resultados das concentrações observadas e simuladas pelo experimento INEL considerando e não considerando o fenômeno de meandro do vento e considerando coeficientes de difusão dados pela equações 9 e 12 . 


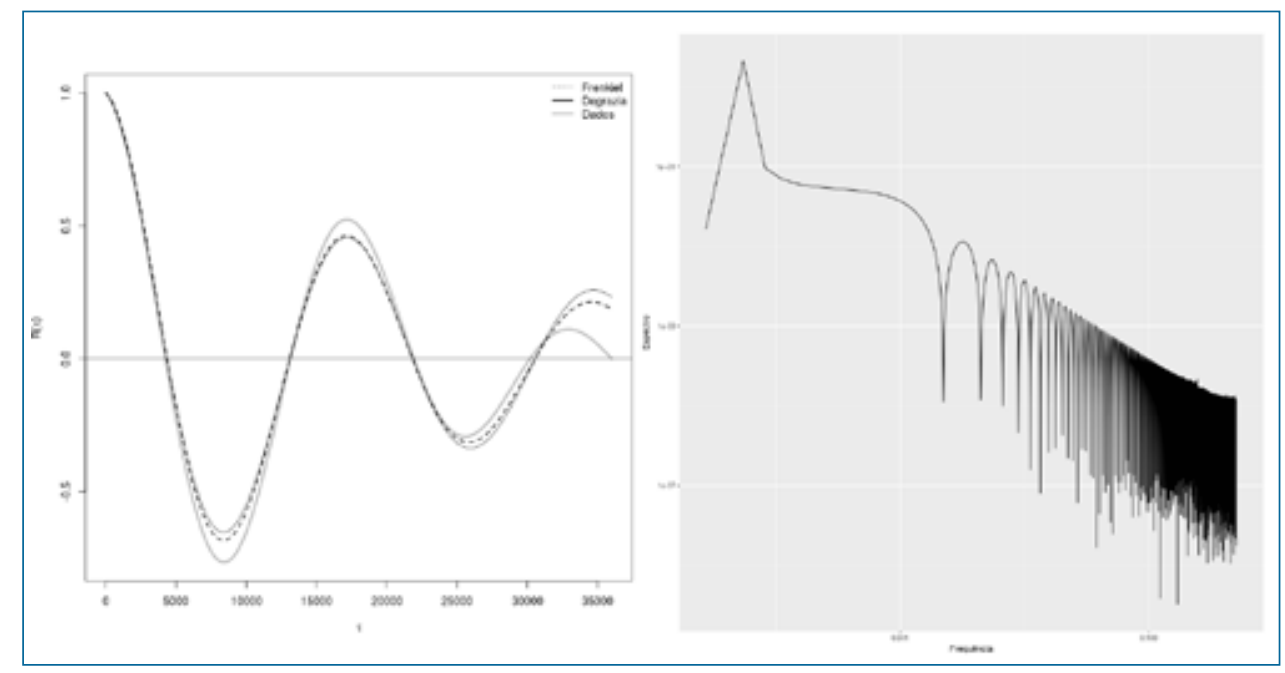

Figura 2: Função de autocorrelação calculada com base na componente u do vento.

Tabela 1: Desempenho estatístico do modelo usando coeficientes de difusão dados pelas equações 9 e 12.

\begin{tabular}{llll}
\hline Simulação & NMSE & COR & FS \\
\hline Meandro - equação 9 & 0,05 & 0,96 & $-0,01$ \\
Sem meandro - equação 9 & 0,11 & 0,93 & $-0,15$ \\
Meandro - equação 12 & 0,16 & 0,92 & 0,05 \\
Sem meandro - equação 12 & 0,18 & 0,89 & $-0,18$ \\
\hline
\end{tabular}

\section{Conclusões}

Descrevemos neste trabalho um método para considerar o fenômeno de meandro do vento em um modelo Euleriano. As funções de autocorrelação apresentam um lóbulo negativo e observa-se através do espectro um pico nas baixas frequências.

O modelo simula de forma satisfatória as concentrações observadas. Os índices estatísticos $N M S E$ e $F S$ são próximos de zero e $C O R$ é próximo de um. Comparando os resultados, considerando e não considerando o meandro do vento (vento dominante da direção x), melhores resultados são obtidos quando o fenômeno de meandro é considerado. O próximo passo deste trabalho é fazer uma análise estatística mais profunda dos resultados.

\section{Referências}

Anfossi, D., Oettl, D., Degrazia, G., Goulart, A. (2005). An analysis of sonic anemometer observations in low wind speed conditions. Boundary-Layer Meteorology, 114, 179-203.

Bates, D., Watts, D. (1988). Nonlinear Regression Analysis and Its Applications. John Wiley \& Sons, New York.

Blackadar, A. (1997). Turbulence and diffusion in the atmosphere: lectures in Environmental Sciences. Springer-Verlag.

Buske, D., Vilhena, M., Moreira, D., Tirabassi, T. (2007). Simulation of pollutant dispersion for low wind conditions in stable and convective planetary boundary layer. Atmospheric Environment, 41, 5496-5501.

Buske, D., Vilhena, M., Tirabassi, T., Bodmann, B. (2012). Air pollution steady-state advection-diffusion equation: the general three-dimensional solution. Journal of Environmental Protection (Print), 3, 1124-1134.

Degrazia, G., Vilhena, M., Moraes, O. (1996). An algebraic expression for the eddy diffusivities in the stable boundary layer: a description of near-source diffusion. Il Nuovo Cimento, 19C, 399-403.

Departamento de computação e matemática (2015). Resolução de sistemas não lineares. URL http: / /wwwp. fc . unesp.br / radriana/Numerico/SNLinear.pdf, acessado em 28 set 2015.

Frenkiel, F. (1953). Turbulent diffusion: mean concentration distribution in a flow field of homogeneous turbulence. Advances in Applied Mechanics, 3, 61-107. 
Hanna, S. (1989). Confidence limit for air quality models as estimated by bootstrap and jacknife resampling methods. Atmospheric Environment, 23, 1385-1395.

Moreira, D., Vilhena, M., Buske, D., Tirabassi, T. (2009). The state-of-art of the giltt method to simulate pollutant dispersion in the atmosphere. Atmospheric Research, 92, 1-17.

Mortarini, L., Anfossi, D. (2015). Proposal of an empirical velocity spectrum formula in low-wind speed conditions. Quarterly Journal of the Royal Meteorological Society, 141, 85-97.

Mortarini, L., Ferrero, E., Falabino, S., Casteli, S., Richiardone, R., Anfossi, D. (2013). Low-frequency processes and turbulence structure in a perturbed boundary layer. Quarterly Journal of the Royal Meteorological Society, 139, 1059-1072.

Panofsky, H., Dutton, J. (1984). Atmospheric Turbulence. John Wiley \& Sons, New York.

Sagendorf, J., Dickson, C. (1974). Diffusion under low wind-speed, inversion conditions. Idaho Falls, Idaho, URL https: //www.arl.noaa.gov/documents/reports/ARL-52.pdf, acesso em 15 jul. 2017.

Sorbjan, Z. (1989). Structure of the atmospheric boundary layer. Prentice Hall, New Jersey.

Szinvelski, C., Degrazia, G., Buligon, L., Moor, L. (2013). Dedução da equação da variança espacial lateral para uma nova formulação da função de autocorrelação lagrangiana. Ciência e Natura, Edição Especial, 187-190.

Viliam Cardoso da Silveira

UFSM - Universidade Federal de Santa Maria E-mail: viliamcardoso@gmail.com

Gervásio Annes Degrazia

UFSM - Universidade Federal de Santa Maria E-mail: gervasiodegrazia@gmail.com

\section{Daniela Buske}

UFPel - Universidade Federal de Pelotas E-mail: danielabuske@gmail.com 